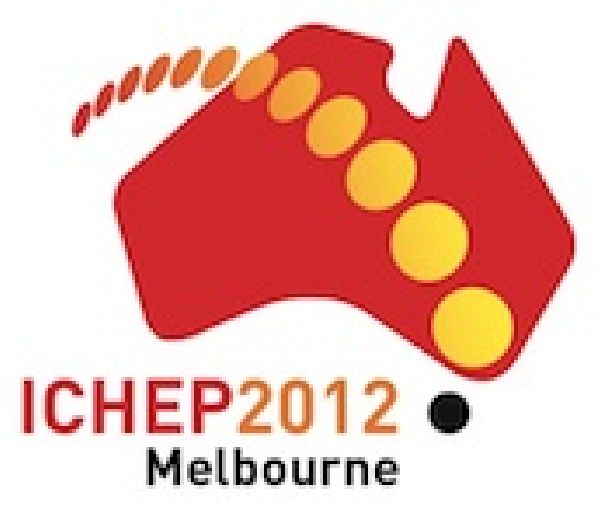

Contribution ID : 554

\title{
The light pseudoscalar meson transition form factor
}

Saturday 07 Jul 2012 at 18:00 (01h00')

\section{Content :}

We use the light fornt quark model in order to improve the theoretical prediction of the transition form factor of $\mathrm{pi}^{\wedge}\{0\}\left(\operatorname{eta}\left({ }^{\prime}\right)\right)$ to gamma ${ }^{\wedge *}$ gamma as a function of the momentum transfer $Q^{\wedge} 2$. We compare our result with the experimental data by BaBar as well as other calculations based on the LFQM. We show that our predicted form factor fits well with the experimental data, particularly those at the large $\mathrm{Q}^{\wedge} 2$ region.

Primary authors : Dr. LIH, Chong Chung (Department of Optometry, Shu-Zen College of Medicine and Management (TW))

Co-authors : Prof. GENG, Chao-Qiang (Department of Physics, National Tsing-Hua University)

Presenter : Dr. LIH, Chong Chung (Department of Optometry, Shu-Zen College of Medicine and Management (TW))

Session classification : Poster Session

Track classification : Track 7. CP Violation, CKM, Rare Decays, Meson Spectroscopy

Type : Poster Sessions 


\title{
The light pseudoscalar meson transition form factor
}

\author{
Chong-Chung $\operatorname{Lih}^{1,2 a}$ and Chao-Qiang Geng ${ }^{3,2 b}$ \\ ${ }^{1}$ Department of Optometry, Shu-Zen College of Medicine \\ and Management, Kaohsiung Hsien, Taiwan 452 \\ ${ }^{2}$ Physics Division, National Center for Theoretical Sciences, Hsinchu, Taiwan 300 \\ ${ }^{3}$ Department of Physics, National Tsing Hua University, Hsinchu, Taiwan 300
}

\begin{abstract}
We use the light front quark model (LFQM) to calculate the transition form factor of $\pi^{0} \rightarrow \gamma \gamma^{*}$ as a function of the momentum transfer $\mathrm{Q}^{2}$. We compare our result with the experimental data by CELLO, CLEO, BaBar and Belle as well as other calculations based on the LFQM. We show that our predicted form factor fits well with the experimental data, particularly those at the large $\mathrm{Q}^{2}$ region.
\end{abstract}

\footnotetext{
${ }^{a}$ E-mail address: cclih@phys.nthu.edu.tw

${ }^{b}$ E-mail address: geng@phys.nthu.edu.tw
} 


\section{INTRODUCTION}

The BaBar and Belle collaborations $[1,2]$ has reported a new data of the $\pi^{0} \rightarrow \gamma^{*} \gamma$ transition form factor $F_{\pi \gamma}\left(Q^{2}\right)$ for the high momentum transfer $Q^{2}$ up to $40 \mathrm{GeV}^{2}$. To describe the data with the $Q^{2}$ dependence, the form factor is fitted to satisfy the formula

$$
Q^{2}\left|F_{\pi \gamma}\left(Q^{2}\right)\right|=A\left(\frac{Q^{2}}{10 G e V^{2}}\right)^{\beta}
$$

with $A=0.182 \pm 0.002 \mathrm{GeV}$ and $\beta=0.25 \pm 0.02$ for the BaBar data. The fitted values from Belle are $A=0.169 \pm 0.006 \mathrm{GeV}$ and $\beta=0.18 \pm 0.05$. Before the BaBar data, most theoretical models predicted that the form factor approaches the QCD asymptotic limit[3], depending on the pion distribution amplitude (DA) with the Q2 dependence under $10 \mathrm{GeV}^{2}[4,5]$. Obviously, the experimental values for $Q^{2}>10 \mathrm{GeV}^{2}$ by BaBar are surprisingly much higher than the QCD asymptotic expectations and thus, cannot be explained by the lowest perturbative results [3]. Even the high order corrections are considered, the large $Q^{2}$ behavior is still hard to be understood. Recently, many proposals have been given in the literature to understand the transition form factor, particularly the BaBar data for $Q^{2}>10 \mathrm{GeV}^{2}$. The Belle result agrees with the previous measurements for $\mathrm{Q}^{2}$ below $9 \mathrm{GeV}^{2}$. In the higher $\mathrm{Q}^{2}$ region, in contrast to BaBar, its results do not show a rapid growth with $\mathrm{Q}^{2}$ and are closer to theoretical expectations[3]. But, the Belle data is also slightly larger than the pQCD asymptotic limit. In this poster, we will use the phenomenological light front (LF) pion wave function to evaluate $\mathrm{Q}^{2}\left|F_{\pi}\left(Q^{2}\right)\right|$ in the light front quark model (LFQM) [7-9]. We will concentrate on the space-like region for the transition form factor. The LF wave function is manifestly boost invariant as it is expressed in terms of the longitudinal momentum fraction and related to transverse momentum variables. The parameter in the hadronic wave function is determined from other information and the meson state of the definite spins can be constructed by the Melosh transformation. We emphasize that our derivation of the form factor can be applied to all allowed kinematic region. In Ref. [10], the study on the transition pion form factor based on the LFQM has been done but the calculation for $\mathrm{Q}^{2}$ was only up to $8 \mathrm{GeV}^{2}$. With the same set of parameters in Ref. [10], the high $\mathrm{Q}^{2}$ experimental data cannot be fitted. The use of the LFQM to understand the BaBar data has been explored in Ref. [11]. However, the conclusion in Ref. [11] has failed to explain the data. In this work, we would like to revisit the LFQM to see if it is indeed the case. 


\section{FRAMEWORK}

The transition form factor of $F_{M \rightarrow \gamma^{*} \gamma^{*}}\left(q_{1}^{2}, q_{2}^{2}\right)$, which describes the vertex of $M \gamma^{*} \gamma^{*}$, is defined by:

$$
A\left(M \rightarrow \gamma^{*}\left(q_{1}, \epsilon_{1}\right) \gamma^{*}\left(q_{2}, \epsilon_{2}\right)\right)=i e^{2} F_{M}\left(q_{1}^{2}, q_{2}^{2}\right) \varepsilon_{\mu \nu \rho \sigma} \epsilon_{1}^{\mu} \epsilon_{2}^{\nu} q_{1}^{\rho} q_{2}^{\sigma}
$$

where $F_{M}\left(q_{1}^{2}, q_{2}^{2}\right)$ is a symmetric function under the interchange of $q_{1}^{2}$ and $q_{2}^{2}$. The light front approach provides a framework for the relativistic quark model in which a consistent and relativistic treatment of quark spins and the center-of-mass motion can be carried out. To find out the transition from factors within the LFQM, We can simplify the mesons wave function into $Q \bar{Q}$ Fock states. Therefore, in the light front (LF) approach, the state wave function can be expressed by an anti-quark $\bar{Q}$ and a quark $Q$ with the total momentum $\mathrm{P}$ as:

$$
\begin{aligned}
&\left|M\left(P, S, S_{z}\right)\right\rangle=\sum_{\lambda_{1} \lambda_{2}} \int[\left.d p_{1}\right]\left[d p_{2}\right] 2(2 \pi)^{3} \delta^{3}\left(P-p_{1}-p_{2}\right) \\
& \times \Phi_{M}^{S S_{z}}\left(x, k_{\perp}\right) b_{\bar{Q}}^{+}\left(p_{1}, \lambda_{1}\right) d_{Q}^{+}\left(p_{2}, \lambda_{2}\right)|0\rangle, \\
& {[d p]=\frac{d p^{+} d^{2} p_{\perp}}{2(2 \pi)^{3}}, }
\end{aligned}
$$

$\Phi_{M}^{\lambda_{1} \lambda_{2}}$ is the amplitude of the corresponding $\bar{q}(q)$ and $p_{1(2)}$ is the on-mass shell LF momentum of the internal quarks. It can be expressed as a covariant form

$$
\begin{aligned}
\Phi_{M}^{S S_{z}}\left(x, k_{\perp}\right) & =\left(\frac{p_{1}^{+} p_{2}^{+}}{2\left[M_{0}^{2}-\left(m_{Q}-m_{\bar{Q}}\right)^{2}\right]}\right)^{\frac{1}{2}} \bar{u}\left(p_{1}, \lambda_{1}\right) \gamma^{5} v\left(p_{2}, \lambda_{2}\right) \phi\left(x, k_{\perp}\right), \\
M_{0}^{2} & =\frac{m_{\bar{Q}}^{2}+k_{\perp}^{2}}{x}+\frac{m_{Q}^{2}+k_{\perp}^{2}}{1-x} .
\end{aligned}
$$

with $\phi\left(x, k_{\perp}\right)$ being the momentum distribution amplitude. In the LFQM, the amplitude disturbation can be solved in principle by the light-front QCD bound state equation. However, we would have to be contented with phenomenological Gaussian type amplitude:

$$
\phi\left(x, k_{\perp}\right)=N \sqrt{\frac{1}{N_{c}} \frac{d k_{z}}{d x}} \exp \left(-\frac{\vec{k}^{2}}{2 \omega_{M}^{2}}\right),
$$


We use a simplest $Q \bar{Q}$ Fock state of the mesons wave function. From the quark-meson loop, we get

$$
\begin{aligned}
A\left(Q \bar{Q} \rightarrow \gamma^{*}\left(q_{1}\right) \gamma^{*}\left(q_{2}\right)\right)= & e_{Q} e_{\bar{Q}} N_{c} \int \frac{d^{4} p_{3}}{(2 \pi)^{4}} \Lambda_{M}\left\{\operatorname { T r } \left[\gamma_{5} \frac{i\left(-\not p_{3}+m_{\bar{Q}}\right)}{p_{3}^{2}-m_{\bar{Q}}^{2}+i \epsilon} \notin_{2} \frac{i\left(\not p_{2}+m_{Q}\right)}{p_{2}^{2}-m_{Q}^{2}+i \epsilon}\right.\right. \\
& \left.\left.\times \notin_{1} \frac{i\left(\not p_{1}+m_{Q}\right)}{p_{1}^{2}-m_{Q}^{2}+i \epsilon}\right]+\left(\epsilon_{1} \leftrightarrow \epsilon_{2}, q_{1} \leftrightarrow q_{2}\right)\right\} \\
& +\left(p_{1(3)} \leftrightarrow p_{3(1)}, m_{Q} \leftrightarrow m_{\bar{Q}}\right),
\end{aligned}
$$

where $N_{c}$ is the number of colors and $\Lambda_{M}$ is a vertex function which related to the monentum

disturbation amplitude of the meson. After integrating over $p_{3}^{-}$and calculated the trace. Here, we have used the definitions of the LF momentum variables and take a frame with the transverse monentum $\left(P-q_{2}\right)_{\perp}=0$ for the $Q \bar{Q}$ state and photon. Thus, the form factor $F_{M \rightarrow \gamma^{*} \gamma^{*}}\left(q_{1}^{2}, q_{2}^{2}\right)$ can be found to be:

$$
\begin{aligned}
F_{M \rightarrow \gamma^{*} \gamma^{*}}\left(q_{1}^{2}, q_{2}^{2}\right)=- & 4 c_{Q}^{2} \sqrt{\frac{N_{c}}{3}} \int \frac{d x d^{2} k_{\perp}}{2(2 \pi)^{3}} \frac{\Phi\left(x, k_{\perp}^{2}\right)}{1-x}\left\{\frac{m_{Q}+(1-x) m_{Q} k_{\perp}^{2} \Theta}{x(1-x) q_{2}^{2}-\left(m_{Q}^{2}+k_{\perp}^{2}\right)}\right. \\
\left.+\left(q_{2} \leftrightarrow q_{1}\right)\right\} & \\
\Phi\left(x, k_{\perp}^{2}\right) & =N \sqrt{\frac{x(1-x)}{2 M_{0}^{2}}} \sqrt{\frac{d k_{x}}{d x}} \exp \left(-\frac{\vec{k}^{2}}{2 \omega_{M}^{2}}\right) \\
\vec{k} & =\left(\vec{k}_{\perp}, \vec{k}_{z}\right), \quad \Theta=\frac{1}{\Phi\left(x, k_{\perp}^{2}\right)} \frac{d \Phi\left(x, k_{\perp}^{2}\right)}{d k_{\perp}^{2}} .
\end{aligned}
$$

The $c_{Q}$ is a factor related to quark charge, $c_{Q}=\frac{2}{3}$ for the up type quark and $c_{Q}=\frac{-1}{3}$ for the down type quark.

\section{NUMERICAL RESULT}

To numerically evaluate the transition form factor of $F_{M \rightarrow \gamma \gamma^{*}}\left(q_{1}^{2}, q_{2}^{2}\right)$, we have to decompose the $\pi^{0}$ meson into $Q \bar{Q}$ Fock states which described as

$$
\left|\pi^{0}\right\rangle=\frac{|u \bar{u}\rangle+|d \bar{d}\rangle}{\sqrt{2}}
$$


If one of $q_{1}$ and $q_{2}$ is on mass shell where the form factors of $F_{M \rightarrow \gamma \gamma^{*}}\left(q_{1}^{2}, q_{2}^{2}\right)$ can be written as

$$
\begin{aligned}
F_{\pi^{0} \rightarrow \gamma \gamma^{*}}\left(Q^{2}\right) & =\frac{4 \sqrt{2}}{3} \sqrt{\frac{N_{c}}{3}} \int \frac{d x d^{2} k_{\perp}}{2(2 \pi)^{3}} \frac{\Phi\left(x, k_{\perp}^{2}\right)}{1-x}\left\{\frac{m_{Q}+(1-x) m_{Q} k_{\perp}^{2} \Theta}{x(1-x) Q^{2}-\left(m_{Q}^{2}+k_{\perp}^{2}\right)}\right. \\
& \left.-\frac{m_{Q}+(1-x) m_{Q} k_{\perp}^{2} \Theta}{\left(m_{Q}^{2}+k_{\perp}^{2}\right)}\right\} .
\end{aligned}
$$

Furthermore, we need to specify the parameters appearing in DA. To constrain the quark masses $\left.m_{(} u, d\right)$ and the meson scale parameters of $\omega$ in DA, we first use its branching ratios of $\pi^{0} \rightarrow 2 \gamma$, given by PDG,

$$
\operatorname{Br}\left(\pi^{0} \rightarrow 2 \gamma\right)=(98.83 \pm 0.034) \%
$$

From

$$
\mathcal{B}\left(\pi^{0} \rightarrow 2 \gamma\right)=\frac{\Gamma\left(\pi^{0} \rightarrow 2 \gamma\right)}{\Gamma}=\frac{(4 \pi \alpha)^{2}}{64 \pi \Gamma_{\pi}} m_{\pi}^{3}\left|F(0,0)_{\pi^{0} \rightarrow 2 \gamma}\right|^{2}
$$

and

$$
f_{\pi^{0}}=4 \frac{\sqrt{N_{c}}}{\sqrt{2}} \int \frac{d x d^{2} k_{\perp}}{2(2 \pi)^{3}} \phi\left(x, k_{\perp}\right) \frac{m}{\sqrt{m_{Q}^{2}+k_{\perp}^{2}}},
$$

we extracte $\left|F(0,0)_{\pi^{0} \rightarrow 2 \gamma}\right|=0.274 G e V^{-1}$. The second condition is the decay constants:

$$
f_{\pi^{0}}=132 \mathrm{MeV}
$$

In ours numerical calculation, the first term in Eq. (11) dominates for the lower region of $\mathrm{Q}^{2}$ and thus, it can be use to describe the experimental data of CLEO and BaBar, with $\mathrm{Q}^{2}$ $\leq 10 \mathrm{GeV}^{2}$. The second one in Eq.(11), related to the non-valence quark contributions, is quite small for a small $\mathrm{Q}^{2}$. Generally speaking this term can be neglected in low $\mathrm{Q}^{2}$ region. But it may enhance the form factor $\mathrm{Q}^{2} F_{\pi}\left(Q^{2}\right)$ with a high value of $\mathrm{Q}^{2}$. As a result, we will include this term in our our numerical calculations. In the Eq. (11), we input the various quark mass within region $m_{q}=0.22 \sim 0.25$ in GeV. Furthermore, we can drived out the meson scale parameters of $\omega$ from Eq.(13) with different quark masses and decay constants. Consequently, we show the $\mathrm{Q}^{2}$ dependence of the $\pi^{0}$ transition form factor with green band in Figs. 1 and 2, respectively. In Fig. 1, we show $\mathrm{Q}^{2} F_{\pi}\left(Q^{2}\right)$ with green band in the high $\mathrm{Q}^{2}$ region. The upper edge of the band is for $m_{q}=0.25$ and $m_{q}=0.22 \mathrm{GeV}$ for lower edge. From the figures, this result can be use to describe the experimental data of Belle. In Fig. 2, 
we concentrate on the behavior of the form factor in the region with $\mathrm{Q}^{2}<10 \mathrm{GeV}^{2}$. In this figure, The upper edge of the band is for $m_{q}=0.25 \mathrm{GeV}$ with $\mathrm{Q}^{2}>4 \mathrm{GeV}^{2}$ and $m_{q}=0.22$ $\mathrm{GeV}$ with $\mathrm{Q}^{2}<4 \mathrm{GeV}^{2}$. It is easy to see that our results fit the data well in the region similar to other theoretical calculations as expected. As we mentioned in the introduction that the BaBar result cannot be fitted by extending the study in Ref.[10] for a high $\mathrm{Q}^{2}$. The main reason is due to the choices of the free parameters, such as the quark masses and $\omega_{\pi}$ leading to a different sharp of the pion wave function. Similarly, the main difference between our results and those in Ref.[11] comes from DAs. It is interesting to point out that our result is almost identical with that in the Regge model [12] and the double logarithmic behavior from the chiral anomaly effects[13].

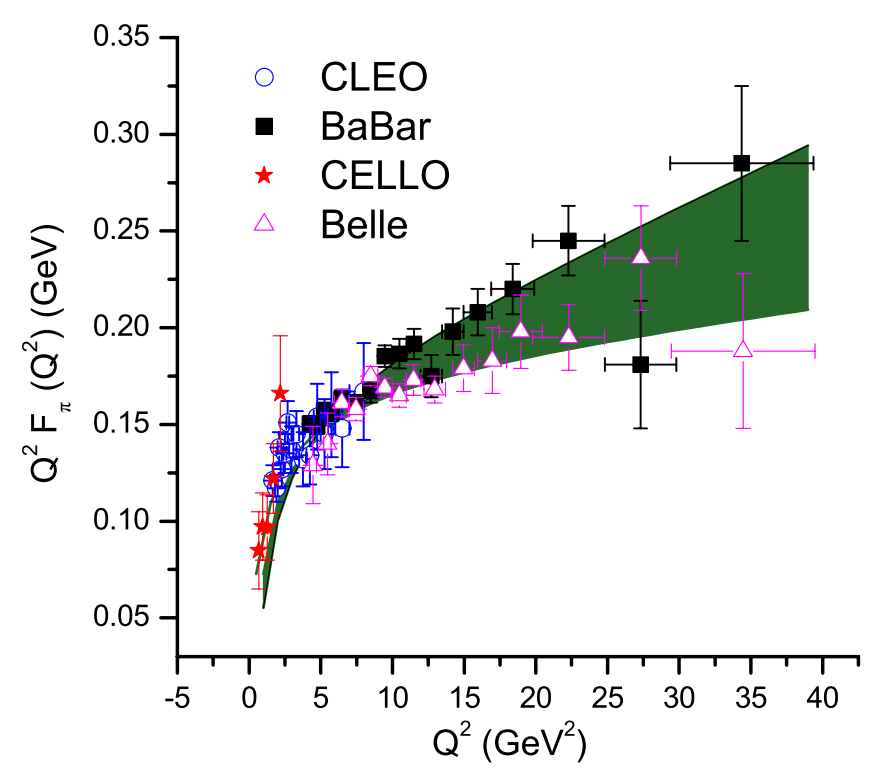

FIG. 1. $\mathrm{Q}^{2}$ dependence of $F_{\pi \gamma}\left(Q^{2}\right)$ in the LFQM.

\section{CONCLUSIONS}

We have studied the form factors of $\pi^{0} \rightarrow \gamma^{*} \gamma$ within the LFQM. In our calculation, we have adopted the Gaussian-type wave function and evaluated the form factors for the momentum dependences in the all allowed $\mathrm{Q}^{2}$ region. We can parametrized the form factor in terms of the double-pole form. Our numerical values are close to the experimental results 


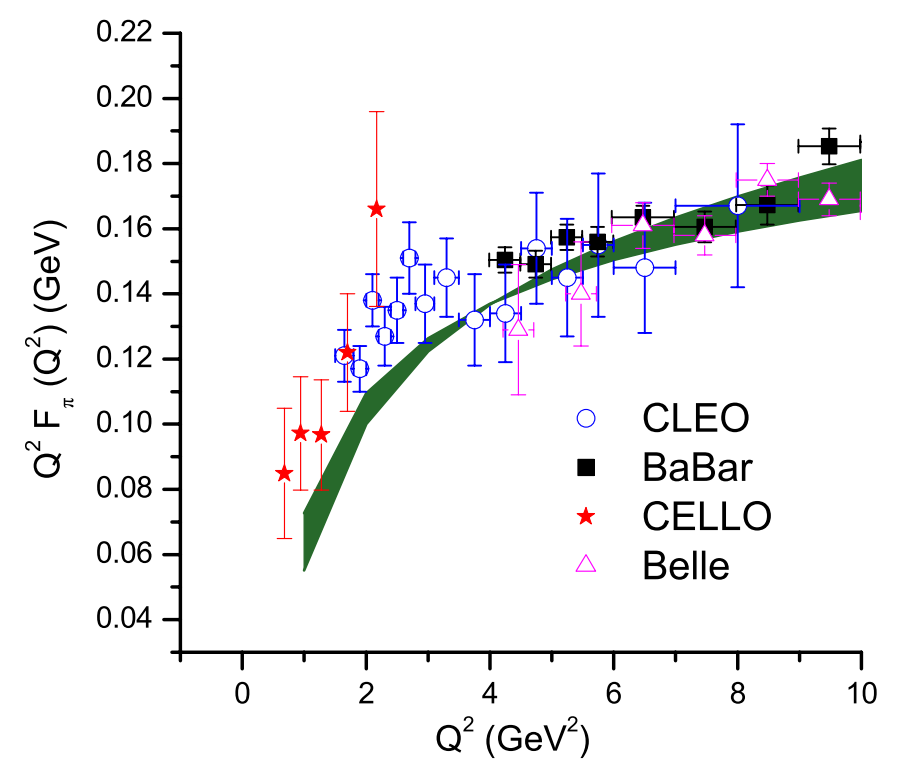

FIG. 2. $\mathrm{Q}^{2}$ dependence of $\left.F_{\pi \gamma}\left(Q^{2}\right)\right)$ in the LFQM for $\mathrm{Q}^{2}<10 \mathrm{GeV}^{2}$.

by BaBar. One can also explain the Belle data well if we used different quark mass. In particular, our results of the transition form factor fit well with the experimental data in the high $\mathrm{Q}^{2}$ region, which cannot be explained in the previous attempts based on the framework of the LFQM. Finally, we remark that due to the large uncertainty in the high $\mathrm{Q}^{2}$ region for the BaBar data, further theoretical studies as well as more precise experimental data are clearly needed.

\section{ACKNOWLEDGMENTS}

This work was partially supported by National Center of Theoretical Science and National Science Council (NSC-97-2112-M-471-002-MY3 and NSC-98-2112-M-007-008-MY3) of R.O.C.

[1] B. Aubert, et al. [The BABAR Collaboration], Phys. Rev. D80, 052002 (2009).

[2] S. Uehara, et al. [The Belle Collaboration], hep-ex/1205.3249.

[3] G. P. Lepage and S. J. Brodsky, Phys. Rev. D22, 2157 (1980). 
[4] G. P. Lepage and S. J. Brodsky, Phys. Lett. B87, 359 (1979).

[5] S. J. Brodsky and G. P. Lepage, Phys. Rev. D24, 1808 (1981).

[6] B. Aubert, et al. [The BABAR Collaboration], Phys. Rev. D74, 012002 (2006); P. del Amo Sanchez, et al. [The BABAR Collaboration], Phys. Rev. D84, 052001 (2011).

[7] C. C. Lih, J. Phys. G38, 065001 (2011).

[8] Chong-Chung Lih and Chao-Qiang Geng, Phys. Rev. C85, 018201 (2012).

[9] C. Q. Geng, C. C. Lih, and C. C. Liu, Phys. Rev. D62, 034019 (2000); C. H. Chen, C. Q. Geng, C. C. Lih, and C. C. Liu, Phys. Rev. D75, 074010 (2007).

[10] Chien-Wen Hwang, Phys. Rev. D64, 034011 (2001).

[11] J. P. B. C. de Melo and T. Frederico, PoSLC 2010, 063 (2010).

[12] W. Broniowski and E. R. Arriola, arXiv:1008.2317; E. R. Arriola and W. Broniowski, Phys. Rev. D81 094021 (2010).

[13] T. N. Pham and X. Y. Pham, Int. J. Mod. Phys. A26, 4125 (2011). 